\title{
A Data-driven load shape profile based building benchmarking: Comparing DOE reference buildings with a large metering dataset
}

\author{
June Young Park ${ }^{1}$, Clayton Miller ${ }^{2}$, Zoltan Nagy ${ }^{1}$ \\ ${ }^{1}$ Intelligent Environments Laboratory, Department of Civil, Architectural \\ and Environmental Engineering, The University of Texas at Austin \\ ${ }^{2}$ Building and Urban Data Science Lab, Department of Building, \\ School of Design and Environment, National University of Singapore
}

\begin{abstract}
There are various sustainable policies or labeling programs in building energy benchmarking. However, they are often oriented for the static information of building performances. The installation of smart metering capabilities with building energy system generates unprecedented amount of data. By analyzing such data, buildings can be evaluated in a more comprehensive manner. We propose a data analytic framework to discover dominant load shape patterns and benchmark buildings with respect to these. We applied this method to a simulation dataset (256 DOE reference building models) and compared them to the results of an actual metering dataset $(3,829$ buildings). Using k-means clustering, we found three fundamental profiles in the actual metering dataset, and two of them, i.e., noon and evening peak profile, are very similar to the dominant load shape patterns of the DOE reference buildings. After regrouping the buildings based on their dominant load shape patterns, we found that $94 \%$ of the buildings are assigned to one of the three fundamental profile shapes (in actual metering dataset), while there were only two groups assigned for the DOE reference buildings. The visual analytics of the proposed benchmarking provides a comprehensive insight on building performance, i.e., not only provides the static information (PSU and EUI) but also temporal aspects (load shape pattern) of building performance.
\end{abstract}

\section{Introduction}

The building sector is one of the main contributors for global energy consumption and greenhouse gas emissions. For example, buildings in the U.S. represent for $40 \%$ of the energy consumption and $38 \%$ of $\mathrm{CO}_{2}$ emissions (DOE (2011)). Therefore, both public and private stakeholders are attempting to develop sustainable policies for a large stock of buildings (Park and Nagy (2018)).

One of the famous sustainable policies in practice is building energy benchmarking (Chung (2011)). When there are a number of buildings to manage, it is important to know whether a specific building in the group performs better or worse than its peer. In this regard, building energy benchmarking helps finding the right peers, i.e., similar buildings in terms of energy performance. One popular benchmarking tool is EPA's Energy Star program. It normalizes energy usage for a variety of factors and it finds the average consumption for a group of input buildings using national survey data. The Energy Star scores based on residuals from ordinary least square regression models and uses the Commercial Building Energy Consumption Survey (CBECS) as a data source to create the peer groups for submitted buildings.

With the importance of benchmarking in building energy policy, then the question is How to group buildings for the right comparison? Traditionally, buildings are classified into man-made categories, e.g., residential, office, classroom, and retail based on their primary space usage (PSU). This classification is the key components when defining the building type, a label that is still actively used in, e.g., CBECS. However, the PSU based classification is inflexible to capture the function of modern buildings, i.e., a whole building does not often exactly fit into these categories due to an increasing diversity of space usages and loads. These buildings are often refereed to as mixed use colloquially, but are often still officially given a rigid building type label. The CBECS data collection protocol instructs that buildings used for more than one of the activities described are assigned to the activity occupying the most floor space. This fuzzy definition creates a situation in which a number of buildings are placed in peer groups that may under or over-estimate their relative energy performance.

In addition to the PSU based classification, current benchmarking methods score buildings according to their energy performance. Energy usage intensity (EUI), which is the annual energy consumption normalized by the gross area of the buildings, is the most widely used in practice due to its convenience of calculation. Although EUI based classification enables users to compare energy performance of building, this metric often also oversimplifies the energetic performance of a building, i.e., EUI information does not capture temporal aspects of energy consumption. 


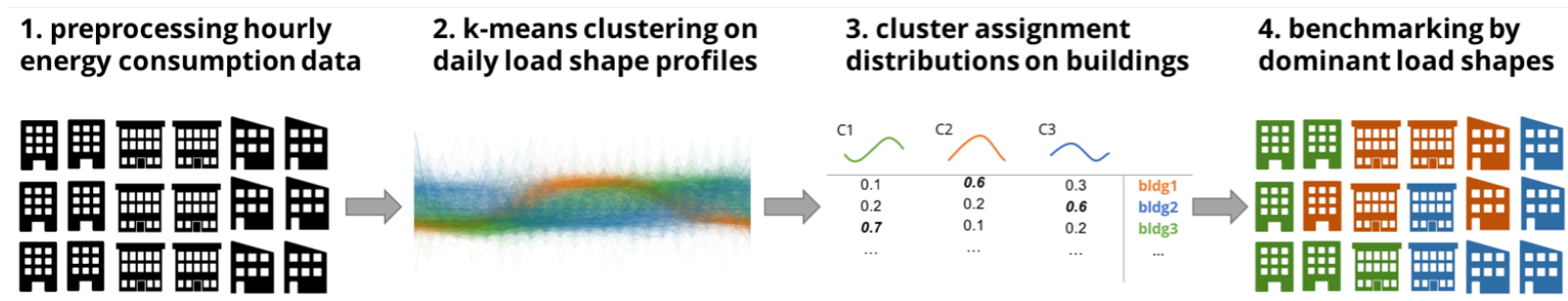

Figure 1: Data analytic framework for benchmarking buildings with respect to their temporal behavior.

The new opportunity has been emerged from the recent technological development in the built environment. For example, researchers are able to understand more comprehensive information on building performance (Gunay et al. (2019)). In particular, smart metering facilities have been widely deployed, e.g., 70 million smart electricity meters were installed in the U.S. by 2016 (EIA (2016)). This integration of smart meters and buildings generates fine-grained energy usage information, and researchers have leveraged this unprecedented increasing smart metering data to analyze building performance more robustly.

The example usages of advanced data analytics in building energy data are described as follow. For a single building, Miller et al. (2015) proposed DayFilter, which detects motifs and discords by analyzing daily energy consumption data using Symbolic Aggregate approximation (SAX). Their tool was further used as fault detection tool for building management system. Using more building data, Miller and Meggers (2017) developed a two step framework to predict various building performance information with a random forest model and applied to 507 non-residential buildings. With even larger scale, Luo et al. (2017) proposed clustering based benchmarking method by analyzing smart meter data from 2,000 commercial buildings in California. Similarly, in our prior work, Park et al. (2019) developed a load shape profile based benchmarking method. We found fundamental load shape profiles and labeled buildings by not man-made artificial labels, e.g., PSU, but rather by their dominant load shape patterns, which is more useful to understand the energetic performance of buildings.

In this paper, we apply a load shape profile based benchmarking to the reference buildings defined by the Department of Energy (DOE) (NREL (2011)). This DOE reference building dataset consists of 16 PSUs and 16 climate zones, i.e., 256 buildings. More importantly, they represent $70 \%$ of the buildings in the U.S. and were successfully used in various building simulation case studies (Field et al. (2010)). With the proposed benchmarking method and reference building models, we questioned 1) whether our benchmarking strategy can classify the reference buildings with respect to their daily energy consumption pattern in order to analyze the buildings within more homogeneous groups, and 2) how the benchmarking re- sult compares to the fundamental profiles found in the real building energy metering dataset. For this comparison, we use our previous results from the largest hourly building electricity consumption dataset (Park et al. (2019)), i.e., 3,829 buildings. Further, we compare both results qualitatively and suggest possible future studies of our work.

The paper is structured as follows. In the Method section, we summarize the data-driven load shape based benchmarking method and introduce the two datasets, i.e., metered and simulated data. Subsequently, the Result section shows both benchmarking results with the discovered profiles. In the Discussion section, we compare the implication of the benchmarking method and both results. The last section concludes the paper.

\section{Method}

\section{Data analytic framework}

Figure 1 illustrates the proposed benchmarking procedure, consisting of four main steps. First, the proposed benchmarking requires hourly energy consumption data for labeling buildings with respect to their energetic behavior. Next, we preprocess the data to limit the number of profiles of each building to a certain year, e.g., 1 or 2 year, in order to ensure that no single building is biasing the clustering result. Subsequently, we eliminate incomplete profiles, i.e., if a daily profile contains less than 24 data points. Lastly, we normalize daily profiles with Z-normalization. Let $\mathrm{t} \in[1,24]$ be the hour of day, and $L_{d}$ is the hourly energy consumption of a building on day $\mathrm{d}$ in $\mathrm{kWh}$. Then, the Z-normalized daily profile is

$$
Z_{d}(t)=\frac{L_{d}(t)-\mu}{\sigma}
$$

where $\mu$ and $\sigma$ are the mean and standard deviation of $L_{d}(t)$. Again, the objective of this benchmarking is discovering load shape pattern, not the magnitude of energy consumption. Particularly, Z-normalization allows us to capture the shape of the profile as the resulting mean for all profiles will be close to 0 , while the standard deviation will be closed to 1 . This normalization technique was successfully implemented in previous related studies (Miller et al. (2015))

Next, we cluster all preprocessed daily profiles. The objective of this clustering step is to investigate the 


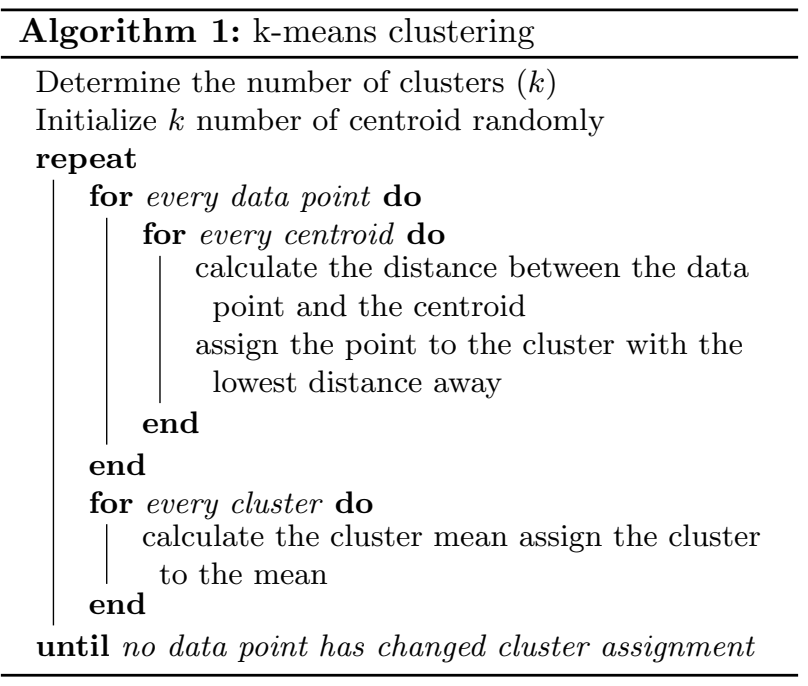

given data points (load profiles) into a certain number of $k$ of clusters that show similarity. These defined clusters are further used as the dominant load shape patterns of benchmarking. We used k-means clustering algorithm (alg. 1). Due to its simplicity, this algorithm has been actively deployed for building energy portfolio and benchmarking studies (Miller et al. (2018)). Although it is popular in the built environment, one challenge of this algorithm is the ambiguity of cluster number, $k$, selection. To decide $k$, we employ two metrics. The first index is the within-cluster sum of squares error (SSE), i.e.,

$$
\mathrm{SSE}=\sum_{i=1}^{k} \sum_{x \in C_{i}}\left(\left\|x-c_{i}\right\|^{2}\right)
$$

where $C_{i}$ is the cluster $i, x$ is a point in cluster $C_{i}$ and $c_{i}$ is the centroid of cluster $C_{i}$. From this indicator, we can evaluate how data points are clustered with shorter distances. In addition, we calculate the Calinski-Harabasz $(\mathrm{CH})$ Score (Caliński and Harabasz (1974)), which offers a trade-off between separation and cohesion by using both the average between and within cluster sum of squares as

$$
\mathrm{CH} \text { Score }=\frac{\sum_{i=1}^{k}\left|C_{i}\left\|\mid c_{i}-c\right\|^{2} /(k-1)\right.}{\sum_{i=1}^{k} \sum_{x \in C_{i}}\left\|x-c_{i}\right\|^{2} /(n-k)}
$$

where $\left|C_{i}\right|$ and $c$ are the number of points in each cluster and the overall centroid of the data, respectively, and $n$ is the number of data points. By analyzing these two metrics, we decide the number of benchmarking groups for the input buildings.

Once the load shape profiles are found from k-means clustering, we re-assemble on a building level and calculate the cluster assignment distribution, i.e., the frequency of each cluster, for each building. To quantify how distinct the load shape profiles of the building are, we calculate the entropy of cluster assignment distribution (Xu et al. (2017)).

$$
E(j)=-\sum_{i=1}^{k} p_{j}\left(C_{i}\right) \log _{2} p_{j}\left(C_{i}\right)
$$

where $p_{j}\left(C_{i}\right)$ is the proportion of cluster type $i$ in building $j$. Larger values for $E(j)$ indicate that various consumption patterns are occurring with similar distribution, i.e., no dominant profiles are present. On the other hand, if $E(j)$ is low, then the building consumed energy in a relatively consistent pattern, i.e., dominant cluster exist. If the same dominant profile is present in a large amount of buildings, it is considered a fundamental load shape profile.

Finally, we label the buildings with their benchmarking groups. In the third step, we identified dominant load shape, i.e., cluster type. Here each dominant load shape serve as benchmarking groups. For this grouping, we define the grouping rule as, i.e.,

$$
G_{k}=\left\{B l d g(j) \mid p_{j}\left(C_{k}\right)>0.5\right\}
$$

If there is no dominant cluster, then these buildings are classified as,

$$
G_{0}=\left\{B l d g(j) \mid p_{j}\left(C_{k}\right)<0.5 \quad \forall k\right\}
$$

where $G_{k}$ and $C_{k}$ are benchmarking group and dominant cluster, respectively. Within the same benchmarking group, their energy consumption patterns are similar so that buildings are compared within the peers of similar energetic behavior.

\section{Actual metering and simulation data}

Again, we apply the proposed benchmarking strategy to the DOE reference building dataset and compare the result with the fundamental profile calculated from one of the largest smart metering datasets (Park et al. (2019)). The main reasons that we employ the DOE reference building in this study are 1) to verify that our benchmarking method can be used for simulation dataset and classify the reference buildings, which are the most representative building models in the U.S., and 2) to compare both actual metering and simulation results in order to investigate the potential improvement of input assumptions to bridge the gap between actual and simulation building performance.

Here, we introduce the input building datasets for both actual smart metering and energy simulation from DOE reference building models. Each dataset is summarized in Tab. 1 and explained as follows.

The first is one of the largest available actual building energy metering datasets (Park et al. (2019)). It is constructed as a single hierarchical data format (HDF5) and consists of multiple sources, which are publicly available for academic purpose. Each individual dataset contains hourly electricity consumption with labeled information, i.e., PSU, building location (city level), and gross area $\left(\mathrm{m}^{2}\right)$. Precisely, this 

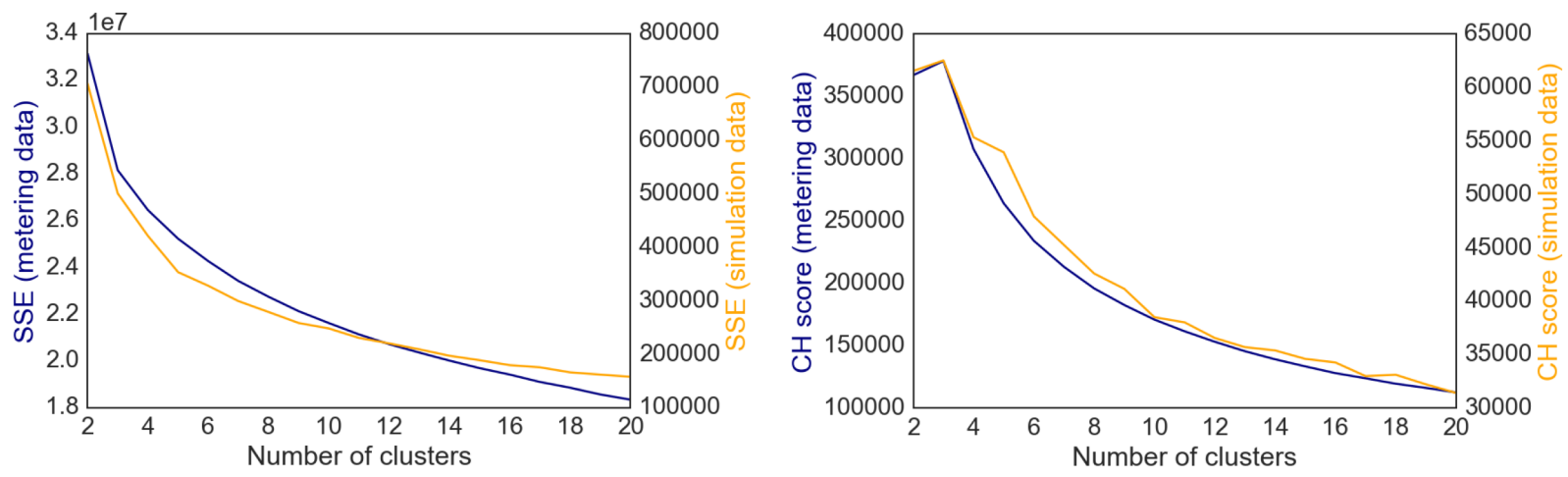

Figure 2: Clustering performance metric result (left: SSE, right: CH score).

Table 1: Summary of actual metering and simulation dataset; More detail descriptions can be found in (Park et al. (2019), NREL (2011)).

\begin{tabular}{rll}
\hline & Metering & Simulation \\
\hline Buildings & 3,829 & 256 \\
Data period & $2 \mathrm{yr}$ & $1 \mathrm{yr}$ \\
Avg. area & $4,709 \mathrm{~m}^{2}$ & $8,573 \mathrm{~m}^{2}$ \\
Avg. EUI & $111 \frac{k W h}{m^{2}}$ & $480 \frac{\mathrm{kWh}}{\mathrm{m}^{2}}$ \\
Climate zones & 5 & 16 \\
PSUs & 75 & 16 \\
\hline
\end{tabular}

dataset follows PSU categories mostly refereed from CBECS survey (EIA (2013)). In brief, the dataset contains hourly energy consumption from 3,829 buildings and 75 PSUs with in average 2 years of collection period.

In addition, we apply this benchmarking strategy to the simulated building energy consumption data. Specifically, DOE reference building models (new construction case) are simulated using EnergyPlus and TMY2 weather files (NREL (2011)). These reference buildings are developed by DOE and associated national laboratories, and formerly called commercial building benchmark models. In total, there are 16 reference building types (PSUs) across 16 locations, resulting in 256 individual building models. Although the actual number of building is less than metering data, these building models are the most representative of the commercial buildings in the U.S. (approximately $70 \%$ ), and the validity of these models has been evidenced by various building simulation studies (Field et al. (2010)).

We described the proposed data-driven benchmarking method and two datasets we tested with. Next section, we analyze the benchmarking results from both datasets.

\section{Results}

\section{Clustering performance index}

The clustering performance indices, i.e., SSE and $\mathrm{CH}$ score are shown in Fig. 2 as a function of $k(k=$
2 -20). In detail, navy and orange colors indicate the results from actual metering dataset and simulation dataset, respectively. Note that the magnitude of each result is significantly different due to the reason that the numbers of buildings are different. Thus, we visualize the results using two separate y-axes. Although the magnitudes of values are different, their patterns are very similar for both datasets, i.e., SSE results continuously decrease with increasing number of clusters, $k$, and $\mathrm{CH}$ scores have a maximum for $k$ $=3$ decrease thereafter.

For further selection of the optimal cluster number $(k)$, we analyzed the SSE plot with elbow method, and found the significant SSE reduction at $k=3$. This is also confirmed by the $\mathrm{CH}$ score, having its maximum values for $k=3$ in both datasets. Thus, from Fig. 2, we select $k=3$ case for further analysis.

\section{Discovered load shape profiles}

With k-means clustering using $k=3$, Fig. 3 (left) illustrates three fundamental profiles from the actual metering dataset, while Fig. 3 (right) indicates the three dominant load shape patterns in DOE reference buildings. The profiles are visualized with three different colors, and $\mathrm{x}$-axis and $\mathrm{y}$-axis indicate time of day and Z-normalized electricity consumption, respectively. In addition, the line thickness is scaled according to the number of profiles for the respective cluster, i.e., a thicker line is indicative of more daily profiles in that particular cluster. Mathematically, these profiles are calculated by cluster centroids, i.e., the mean values of the profiles within associated cluster.

We discovered similar load shape patterns from both actual metering (3,829 buildings) and simulation (256 DOE reference buildings). As shown in Fig. 3, green and blue load shape pattern illustrate noon and evening peak profile, respectively, in both datasets. The minor difference is that the profiles from metering data shows smoother shape with shorter peak time and smaller variations in terms of magnitude of Z-normalized load. In addition, both green and blue profiles are the thickest profiles, indicating most of daily profiles in both datasets are noon and evening 

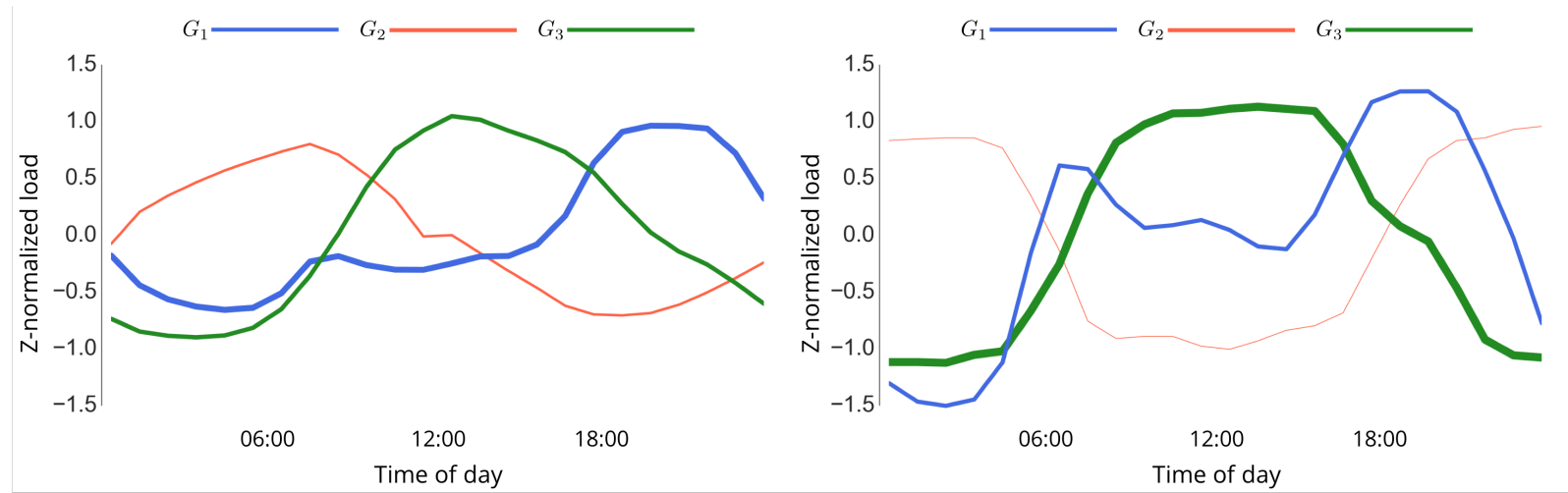

Figure 3: Discovered fundamental profiles (left: metering data, right: simulation data).

Table 2: Number of buildings and average entropy (G1: blue load shape, G2: red, and G3: green).

\begin{tabular}{lllll}
\hline & \multicolumn{3}{c}{ Metering } & \multicolumn{2}{c}{ Simulation } \\
& $\begin{array}{l}\text { Nr. } \\
\text { bldgs }\end{array}$ & $\begin{array}{l}\text { Avg. en- } \\
\text { tropy }\end{array}$ & $\begin{array}{l}\text { Nr. } \\
\text { bldgs }\end{array}$ & $\begin{array}{l}\text { Avg. en- } \\
\text { tropy }\end{array}$ \\
\hline$G_{0}$ & 247 & 1.5 & 0 & - \\
$G_{1}$ & 1,868 & 1.0 & 80 & 0.05 \\
$G_{2}$ & 561 & 0.75 & 0 & - \\
$G_{3}$ & 1,153 & 0.95 & 176 & 0.4 \\
\hline
\end{tabular}

peak load shapes primarily.

On the other hand, red profiles represent totally different load shape patterns for each dataset. In left part of Fig. 3 (actual metering dataset), red load shape indicates the maximum and minimum consumption at morning and evening, respectively. Differently, we observed that red profile has low and steady consumption during noon period from simulation data. However, red profiles are the thinnest profiles, which means that there are only a few daily profiles behaving like red load shape patterns in both datasets.

\section{Regrouping by cluster dominance}

The buildings are then regrouped with respect to dominant cluster types. Tab. 2 represents the regrouping result based on each building's dominant energy consumption pattern. From the metering dataset, only $6 \%$ (247) of buildings are classified as $G_{0}$, which indicates that total $94 \%$ of buildings have a dominant load shape and are grouped accordingly. In addition, the entropy value of $G_{0}$ is relatively high, which also confirms that there is no such dominance energy consumption pattern for these 247 buildings. On the other hand, the major portions of the buildings are grouped as $G_{1}(48 \%), G_{2}(16 \%)$ and $G_{3}$ $(30 \%)$ with relatively low entropy values. This observation suggests that the majority of the buildings have their dominant load shape patterns and those load shapes are fairly consistent based on their smart metering records.
For the simulation dataset, on the other hand, none of the buildings are classified as $G_{0}$ and $G_{2}$. About $30 \%$ of the buildings are part of $G_{1}$ and the averaged entropy values within this group is extremely low. This suggests that 80 DOE reference buildings are dominantly behaving as blue load shape, i.e., evening peak profiles. The other $70 \%$ of the buildings are grouped as $G_{3}$. Again the averaged entropy values of this group is small enough to argue that these 176 buildings have a dominant load shape pattern as green profile in Fig.3 (right). In general, the entropy values of simulation dataset is lower than one from actual metering dataset. This is because, simulation results are calculated predominantly by input assumptions and weather files, whereas metering dataset has some uncertainties regarding building system performance, occupant behavior, and inconsistent weather conditions.

\section{Analyzing building performance with PSU, EUI, and dominant load shape profile}

Fig. 4 illustrates the final benchmarking result with dominant load shape profiles. For the metering dataset, we randomly sampled 256 buildings from the whole dataset (3,829 buildings) for visualization purpose, on the other hand, we visualize all 256 buildings for simulation dataset. The buildings are grouped according to $G_{0}-G_{3}$, each dot representing one building colored according to its PSU for reference and EUI values on y-axis. Such visual analytic provides multidimensional insights on building performance, i.e., analyzing PSU, EUI, and dominant load shape profile. As an example, even though two classroom buildings in actual metering dataset are same PSU and EUI, we can differentiate by their different energy consumption pattern (G2 or G3) with this result.

Regarding PSUs, buildings from actual metering analysis are classified for all groups $\left(G 0-G_{4}\right)$. Mainly, $G_{1}$ contains single family house type buildings, while other groups have various PSUs. This indicates that blue load shape pattern in Fig. 3 is derived by residential building. Conversely, other load shape patterns are independent from PSU types. For 

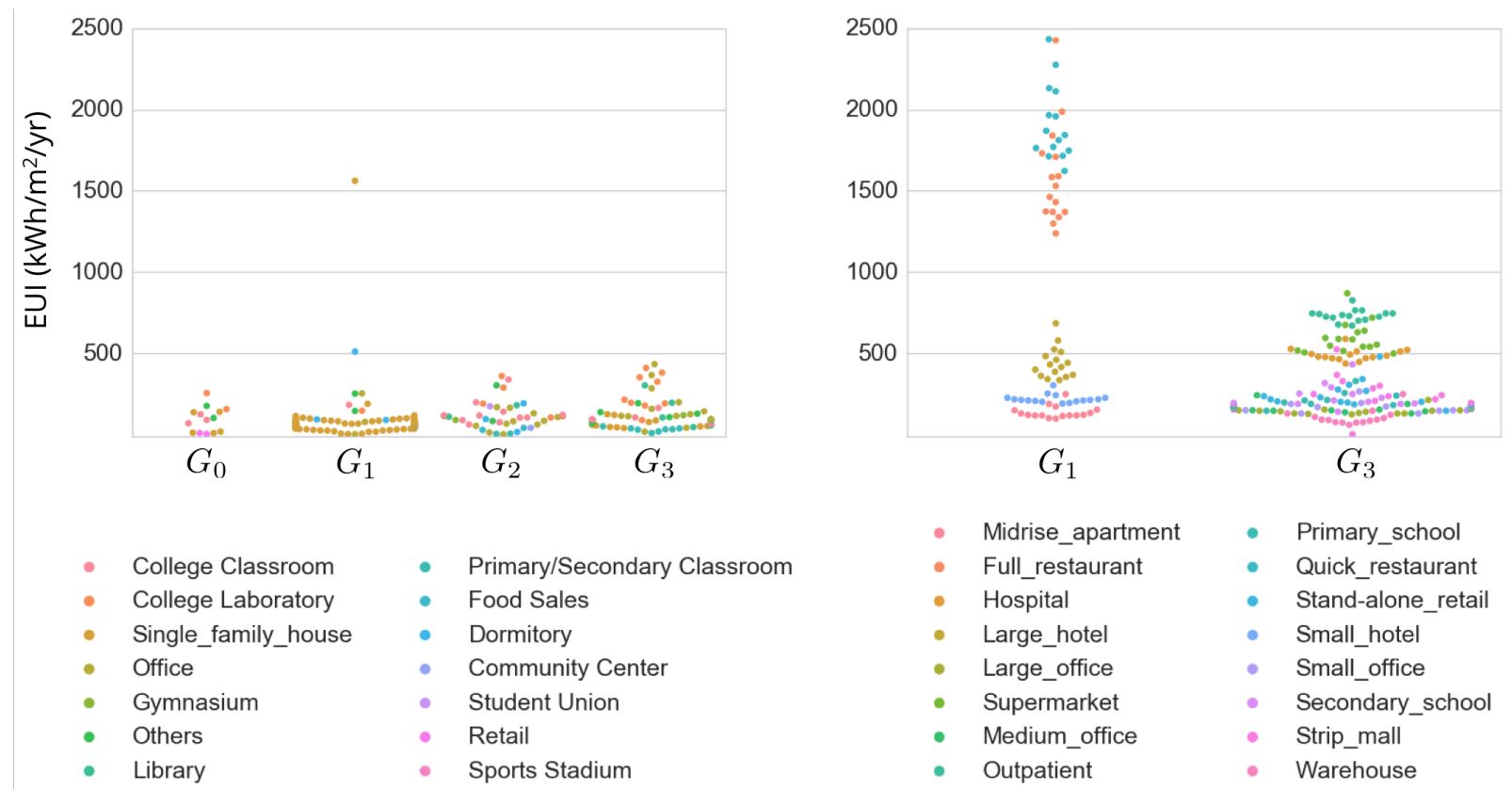

$\begin{array}{ll}\text { - } & \text { Primary/Secondary Classroom } \\ \text { - } & \text { Dood Sales } \\ \text { - } & \text { Commitory } \\ \text { - Student Union } \\ \text { - Retail } \\ \text { - Sports Stadium }\end{array}$

$\begin{array}{ll}\text { - } & \text { Midrise_apartment } \\ \text { - } & \text { Hosll_restaurant } \\ \text { - } & \text { Large_hotel } \\ \text { - } & \text { Large_office } \\ \text { - Supermarket } \\ \text { - } \\ \text { - } \text { Medium_office } \\ \text { Outpatient }\end{array}$

- Primary_school
- - Stand-restaurant
- Small_hotel
- Small_office
- Secondary_school
- Strip_mall
- Warehouse

Figure 4: Dominant load shape profile based benchmarking result (left: metering data, right: simulation data).

simulation dataset, $G_{1}$ and $G_{3}$ are separated completely, i.e., $G_{1}$ has midrise apartment, large / small hotel, and full / quick restaurant, and $G_{3}$ has other PSUs. This observation suggests that load shape pattern is dependent on schedule inputs of reference building models.

In addition, this visual analytic tool quickly returns the building performance overview of a large amount of buildings. For example, more than half of the buildings from metering dataset are grouped in $G_{1}$, which indicates that the grid needs to be robust enough to maintain this demand pattern, i.e., evening peak. Also, the buildings in $G_{0}$ might have malfunctioning building systems or metering infrastructures. Thus, this grouping could serve as a preliminary step in automated fault detection.

\section{Discussion}

The proposed benchmarking method regrouped a large amount of buildings for both actual metering and simulation dataset according to their dominant load shape patterns. Each group has unique load shape pattern and indicates low entropy value, which means these profiles are unique characteristics of buildings. This result suggests that when building researchers or policy makers analyze temporal aspects of building performance, a load shape pattern based benchmarking only requires $2-4$ groups for a large stock of buildings whereas typically $20-50$ individual PSU in a conventional benchmarking strategy.

Interestingly, two of the fundamental profiles from actual metering are similar to two dominant load shape patterns from DOE reference building simulation dataset, i.e., blue and green load shape pat- tern. First, blue load shape pattern is representing small and large peak at morning and evening, respectively. The major contributions for this type of profile are residential buildings in metering dataset. Similarly, in simulation dataset, all of the residential and hotel buildings have blue load shape pattern as dominant cluster. In addition, green load shape patterns from actual metering and simulation dataset are similar, i.e., peak time around noon, and various building PSUs derive this load shape profile. Lastly, even though red load shape patterns are completely different each other, these profiles again are not the main load shape patterns in both datasets, i.e., $16 \%$ of the buildings in metering dataset and none of the DOE reference buildings have red load shape as dominant cluster. This observation suggests that DOE reference building models capture real world building cases at least for dominant building load shape patterns.

On the other hand, the main difference between the two results is that DOE reference buildings are only grouped by 2 dominant groups while buildings from actual dataset are classified with all 4 groups including no-dominance group $\left(G_{0}\right)$. This is mainly because, simulation result from DOE reference buildings are governed by modeling assumptions. For example, Fig. 5 indicates modeling assumptions, i.e., building occupancy, equipment, light schedules, for DOE reference building models. As we described, these models have 16 different PSUs. Although all 16 occupancy profiles and schedules are different in terms of numbers, some PSUs do not generate significantly different energy simulation results, e.g., the pairs of restaurants, hotels, offices, and store buildings. Such similar inputs are the main reason for 


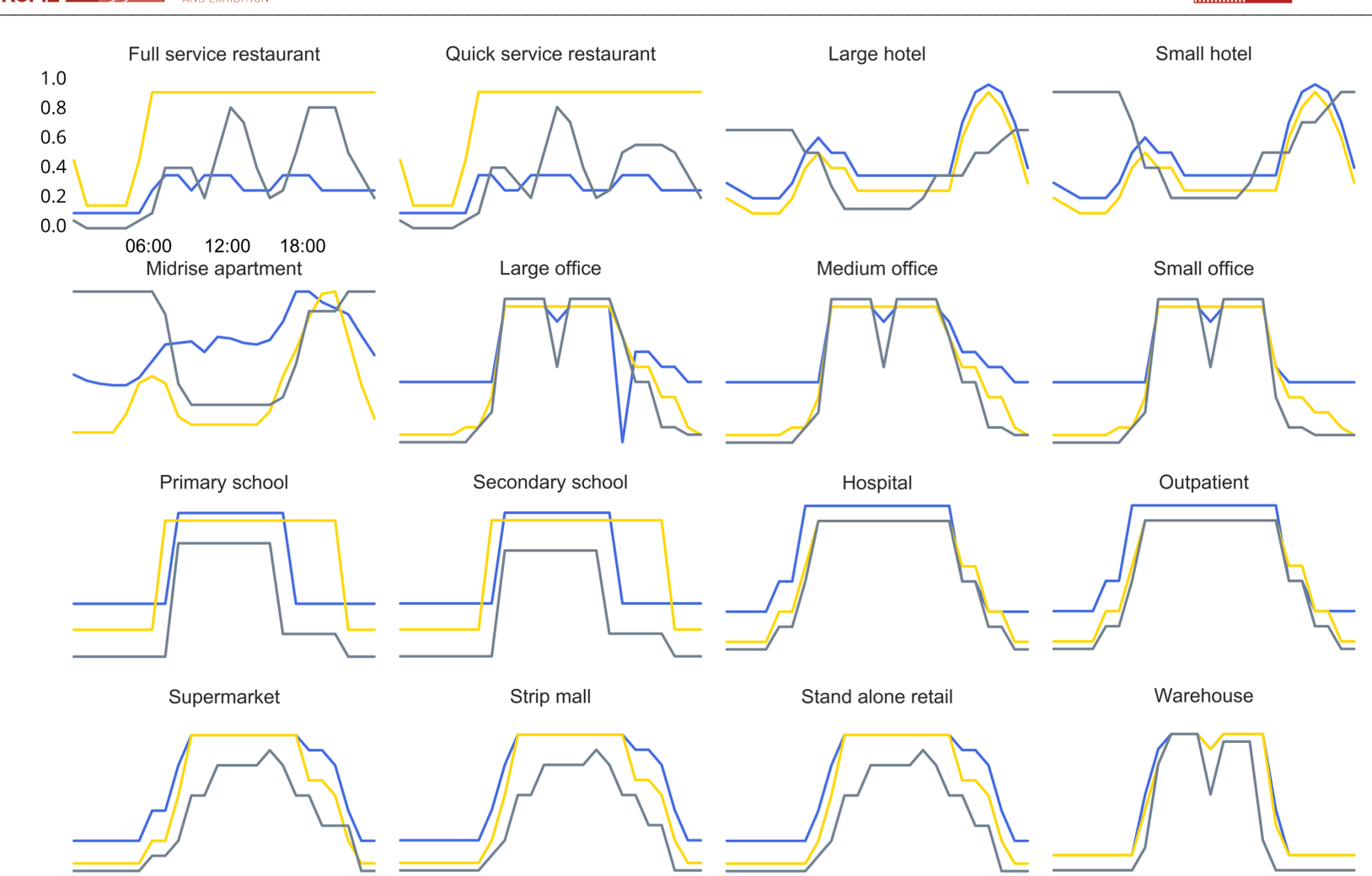

Figure 5: Modeling assumptions for DOE reference buildings (grey: occupancy, blue: equipment, and yellow: light schedule).

generating only two benchmarking groups in Fig. 2. In fact, researchers tried to simulate building performance with more accurate input assumptions (Yan et al. (2017)). As a future work, we can integrate the DOE reference building models with some occupant behavior models from previous literature (Chen et al. (2018)) in order to have more realistic result.

As shown in Fig. 3 (right), three dominant profiles from simulation dataset are highly related to input assumptions in Fig. 5. Thus, we can reversely infer occupancy profiles and building system operation schedules from the hourly energy consumption data. Since the actual metering dataset, which we used in this paper, is one of the largest datasets (hour resolution energy consumption from 3,829 buildings), we can potentially calculate very realistic temporal input assumptions for future reference building models.

Although our proposed method was successfully implemented for both actual metering and simulation dataset, it should be noted that the results are still carefully examined in terms of its ability to generalize to all locations and all building types. This type of limitation is common in any data-driven approach. Especially, the actual metering dataset mainly contains educational buildings from North America and residential buildings from Europe (Cork/Ireland), which might result in similar predefined building operation schedule and occupant behavior. In addition, as we stated above, DOE reference building models are not exhaustive to capture various occupant behaviors and building schedules in real buildings. Lastly, we can further improve the simulation result by changing weather files.

\section{Conclusion}

In this paper, we investigated an interesting temporal aspects, i.e., dominant load shape pattern, of building performance using unsupervised machine learning methods, and applied them to a data-driven benchmarking study. We applied this method to the simulation dataset (256 DOE reference buildings) and compared it to the result from an actual metering dataset (3,829 buildings). With k-means clustering method, $k=3$ was selected as the optimal number of groups for both dataset. As a result, we discovered three fundamental profiles from the actual metering dataset, and two of them, i.e., noon and evening peak profile, are very similar to the dominant profiles from DOE reference buildings. We then regrouped the buildings according to their dominant load shape pattern. For the actual metering dataset, we found that $94 \%$ of the buildings are assigned to one of the three fundamental profile shapes, while there were only two groups assigned for the DOE reference buildings. Finally, we visualized the load shape profile based benchmarking result with a conventional way of building classification, i.e., PSU and EUI based. This novel benchmarking approach has evidenced its potential applications for more robust building data analytics. 


\section{References}

Caliński, T. and J. Harabasz (1974). A dendrite method for cluster analysis. Communications in Statistics-theory and Methods 3(1), 1-27.

Chen, Y., T. Hong, and X. Luo (2018). An agentbased stochastic occupancy simulator. In Building simulation, Volume 11, pp. 37-49. Springer.

Chung, W. (2011). Review of building energy-use performance benchmarking methodologies. Applied Energy 88(5), 1470-1479.

Energy Efficiency \& Renewable Energy Department (2011). Building energy databook.

U.S. Energy Information Administration (2013). Commercial buildings energy consumption survey (CBECS) - building type definitions.

U.S. Energy Information Administration (2016). How many smart meters are installed in the united states, and who has them?.

Field, K., M. Deru, and D. Studer (2010). Using doe commercial reference buildings for simulation studies. Proceedings of SimBuild 4(1), 85-93.

Gunay, H. B., W. Shen, and G. Newsham (2019). Data analytics to improve building performance: A critical review. Automation in Construction 97, 96-109.

Luo, X., T. Hong, Y. Chen, and M. A. Piette (2017). Electric load shape benchmarking for small-and medium-sized commercial buildings. Applied Energy 204, 715-725.

Miller, C. and F. Meggers (2017). Mining electrical meter data to predict principal building use, performance class, and operations strategy for hundreds of non-residential buildings. Energy and Buildings $156,360-373$.

Miller, C., Z. Nagy, and A. Schlueter (2015). Automated daily pattern filtering of measured building performance data. Automation in Construction 49, $1-17$.

Miller, C., Z. Nagy, and A. Schlueter (2018). A review of unsupervised statistical learning and visual analytics techniques applied to performance analysis of non-residential buildings. Renewable and Sustainable Energy Reviews 81, 1365-1377.

National Renewable Energy Laboratory (2011). U.S. Department of Energy Commercial Reference Building Models of the National Building Stock.

Park, J. Y. and Z. Nagy (2018). Comprehensive analysis of the relationship between thermal comfort and building control research-a data-driven literature review. Renewable and Sustainable Energy Reviews 82, 2664-2679.
Park, J. Y., X. Yang, C. Miller, P. Arjunan, and Z. Nagy (2019). Apples or oranges? identification of fundamental load shape profiles for benchmarking buildings using a large and diverse dataset. $A p$ plied Energy 236, 1280-1295.

Xu, S., E. Barbour, and M. C. González (2017). Household segmentation by load shape and daily consumption. Proc. 6th ACM SIGKDD Int. Worksh. Urban Computing 2.

Yan, D., T. Hong, B. Dong, A. Mahdavi, S. DOca, I. Gaetani, and X. Feng (2017). Iea ebc annex 66: Definition and simulation of occupant behavior in buildings. Energy and Buildings 156, 258-270. 